\title{
Valorization of wine making by-products
}

New uses: additives, cosmetics, pharmacy...

\section{OBJECTIVES}

$\checkmark$ Demonstrate the economic potential and ecological of wine by-products with a bibliographic research.

$\checkmark$ Gain deep knowledge in resveratrol and its posssible benefits in the organism.

Evaluate the European and American status of the resveratrol as an ingredient.

\section{BY-PRODUCTS}

$\bigcirc$ Stalks and pomace $(12,5 \%) \rightarrow$ animal feed, additives, grappa, biofuel, energy

O Grape seeds $(20 \sim 30 \%) \rightarrow$ additives, grape seed oil, biofuel

$\circ \quad$ Yeast lees $\rightarrow$ pigments, $\boldsymbol{\beta}$-glucan, citric acid

\section{EFSA Status}

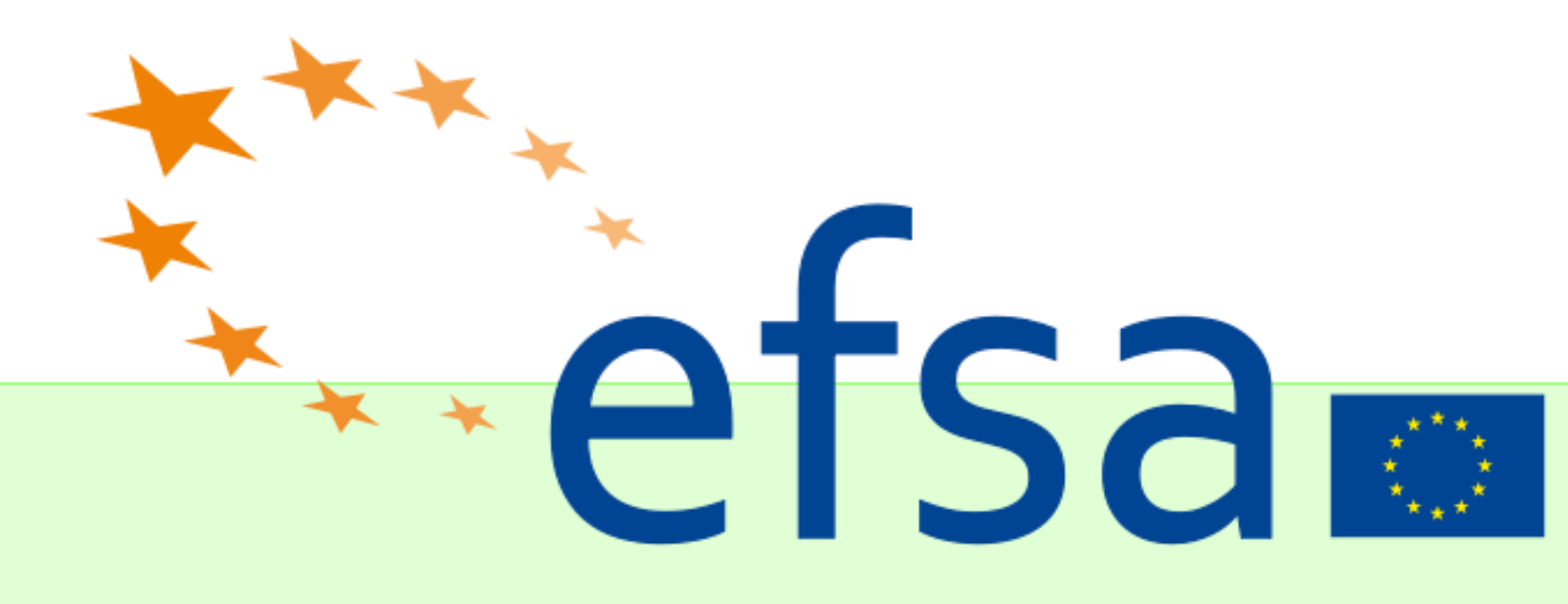

European Food Safety Authority

DSM sent a consult regarding the safety of synthetic trans-resveratrol.

Consult $\rightarrow \checkmark$ NOAEL:750 mg/kg day $/ \mathrm{bw}$

\section{RESVERATROL}

- Polyphenolic compound $\longrightarrow$ stilbene

- Present in grapes, peanuts and red fruits

- Demonstrated Organism Effects: Antioxidative, LDL $\downarrow$, cancer prevention, cardiovascular disease reduction.

- Economic potential of $40 M €$

\section{FDA Status}

GRAS since 2007 in bottled water. Max. Concentration I $10 \mathrm{mg} / \mathrm{L}$

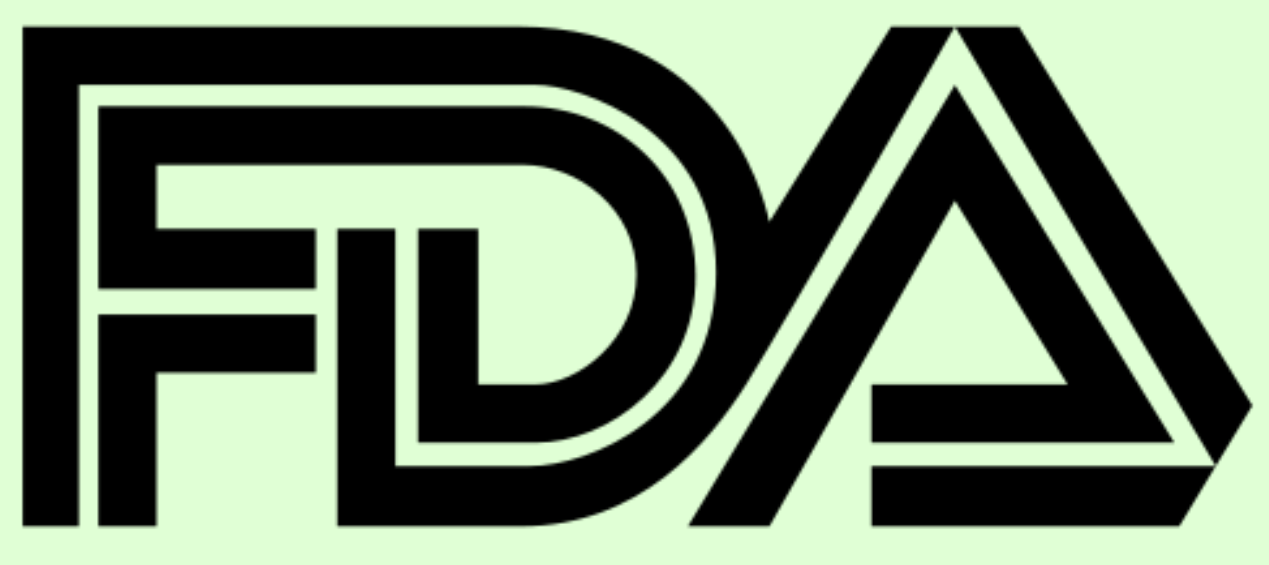

\section{Conclusions:}

- By-product valorization is a high potential process.

- Most of resveratrol benefits have not been demonstrated with solid evidence.

- Even though wine by-products receive a valorization it is important to achieve more profitable processes.

- Wine by-products valorization as well as other by-products is an effective way to maximize its potential. 